\title{
Radiative Transfer Modeling of Simulation and Observational Data
}

\author{
Jürgen Steinacker ${ }^{1}$, Thomas Henning ${ }^{1}$ \& Aurore Bacmann ${ }^{2}$ \\ ${ }^{1}$ Max-Planck-Institut für Astronomie, Königsstuhl 17, 69117 Heidelberg, Germany \\ email: stein@mpia.de, henning@mpia.de \\ ${ }^{2}$ Université Joseph Fourier - Grenoble 1/CNRS, Laboratoire d'Astrophysique de Grenoble \\ (LAOG) UMR 5571, BP 53, 38041 Grenoble Cedex 09, France \\ email: aurore.bacmann@obs.ujf-grenoble.fr
}

\begin{abstract}
Radiative Transfer (RT) is considered to be one of the four Grand Challenges in Computational Astrophysics aside of Astrophysical Fluid Dynamics, N-Body Problems in Astrophysics, and Relativistic Astrophysics. The high dimensionality (7D instead of 4D for MHD) and the underlying integro-differential transport equation have forced coders to implement approximative RT methods in order to fit spectra and images or to treat RT in their HD and MHD codes.

The central role of RT in star formation (SF) is based on several facts: a) The dense dusty gas in $\mathrm{SF}$ regions alters the radiation substantially making $\mathrm{SF}$ one of the most complex applications of RT. b) Radiation transports energy within the object and is therefore an essential part of any dynamical SF model. c) RT calculations tell us which of the processes/structures are visible at what wavelength by which telescope/instrument. Hence, RT is the central tool to analyze simulation results or to explore the scientific capabilities of planned instruments. d) With inverse RT, we can obtain the 1D-3D density and temperature structure from observations, completely decoupled from any (M)HD modeling (and the approximations made within).

In this review, we summarize the main difficulties and the currently used computational techniques to calculate the RT in SF regions. Recent applications of 3D continuum RT in molecular clouds and disks around young massive stars are discussed to illustrate the capabilities and limits of current RT modeling.
\end{abstract}

Keywords. accretion, accretion disks, radiative transfer, scattering, methods: data analysis, methods: numerical, stars: formation, ISM: clouds, ISM: dust, extinction, ISM: globules, infrared: ISM

\section{Introduction}

Since scientist have started to tackle the problem of star formation with the aid of computational facilities, the two main questions are: a) Which are the essential physical processes we have to model? and b) which processes can we afford to model given the current computer power? As illustrated in the historical part of these proceedings, the goal of the computational models run on older computers was to understand the combined action of the three basic processes gravitation, gas kinematics, and magnetic fields (see, e.g., the review by Norman). With the use of adaptive meshes and smooth particle codes it became possible to handle the six orders-of-magnitude variation in density as documented in the method section. More recently, codes have been able to study special aspects of the three basic processes, namely jets as a combined action of gas kinematics and magnetic fields, winds, or turbulence within the potentially magnetized gas (see star formation feedback session). Meanwhile, it is also considered to include basic chemical networks in order to determine the ionization degree of the gas for magnetic field coupling purposes, or 
to compute the abundances of molecules with observable line transitions. While chemical network solvers are no computational problem nowadays, their consideration slows down the codes depending on the sophistication of the network.

In this perspective, the inclusion of radiative transfer effects appears to be a further refinement after the major ingredients to form a star have been dealt with. This is by far not the case, and this misleading concept has been discussed controversially during the conference.

Radiation is a basic ingredient of the star formation process. It is due to its high dimensionality that it was neglected in early calculations or crudely approximated by some heating/cooling functions. With the currently available computer power, the star formation community now is starting to return to more realistic descriptions of how the radiation is transported within the gas, as discussed in this book in the radiative dynamics and feedback sessions. For example, the question where and when gas is starting to collapse to form protostars strongly dependents on a correct treatment of the cooling. In the dense regions of cloud cores, the cooling is dominated by the dust, and it takes complex radiative transfer calculations to determine the heating and cooling in each cell of the computational grid.

There are two main obstacles when performing RT calculations. The intensity of the 3D radiation field depends on the wavelength, three spatial, two directional coordinates, and time. To get an impression of the required resolution, one can assume a decent resolution of 100 points in each variable (or the equivalent number of spatial grid cells when using adaptive grids), In this case, the solution vector has $10^{14}$ entries. Beside this enormous requirement for the internal memory size of the used computer, the RT equation is an integro-differential equation including a scattering integral, making it difficult to apply common solvers. And 100 grid points might not be enough depending on the problem, e.g., to resolve the strongly peaked UV radiation of the stars in the direction space. Steinacker et al. (1996) calculated optimized equally-spaced direction grid points on the unit sphere, but still $100 \times 100$ directional grid points correspond to a mean resolution of about $2.7^{\circ}$ only.

Aside of its role to transport energy within the object, the transport of the radiation from the enshrouded object to the observer is one of the most fundamental processes in astrophysics, and crucial for our interpretation whenever radiation is altered on its way from the source to the telescope. Within computational star formation, it remains the greatest challenge to link the observational data to the simulation findings. The alteration of the radiation can range from small perturbations like the reddening of stellar light due to interstellar extinction or the change of polarization due to the Faraday effect, to almost complete shielding at short wavelengths and thermal re-emission at infrared and sub-millimeter wavelengths in the case of deeply-embedded star-forming regions.

\section{Radiative transfer: solvers and improvements}

Comparing continuum and line radiative transfer. For the physical interpretation of observed images, often both sets of line and continuum data have to be considered simultaneously. Here, line transfer is numerically more complex as well as in terms of modeling.

Line RT generally has to be performed for atomic or molecular effects of photon absorption, scattering, or emission. As the atoms and molecules are moving and the lines appear in a narrow wavelengths range, the emission is strongly influenced by the velocity field within the astrophysical structure to investigate. Furthermore, the abundance of the atom or molecule within the gas has to be known or to be determined often depending 
on a complex network of chemical reactions. This has several effects on calculating and modeling with line RT. First, parameter numbers of the order of several hundreds are needed to describe the model of the gas in its morphology, chemical composition, and kinematics. As the images and SEDs contain line-of-sight-integrated information only, modeling line data means to deal with possible model ambiguities. The model parameters are also strongly coupled so that it is hard to disentangle them just from the line data. On the other side, there is hope the richness of the data will enable us to improve our understanding of the astrophysical processes substantially once 3D line transfer codes are able to determine all parameters. Second, to know the emission of a cell of gas, the level population of the molecule needs to be determined. Given the often complex level structure of the important molecules, this will slow down the line RT calculation (e.g. compared to continuum codes by a factor 10 to 100). Thirdly, the Doppler shift term appears in the equation system which is not present in the continuum case.

Continuum RT is considered, e.g., for dust particles as small solid bodies which absorb, scatter, and re-emit radiation. The micron-sized particles which commonly dominate the RT are well-mixed in the gas, and follow a size and shape distribution (Min et al. 2003) as well as a distribution of chemical compositions. This again introduces a strong ambiguity in the modeling process of projected images and SEDs. The time-dependent re-emission of smaller particles down to a size of a few atomic layers again requires more computational effort to calculate the local mean dust temperature (Guhathakurta \& Draine(1989) or Siebenmorgen et al. (1992)).

In the following, we will concentrate on the simple case of continuum RT of dust particles which have the size of typical interstellar dust particles around the tenth of a micron.

Solution methods. Each of the solution algorithms used sofar has its advantages and drawbacks.

In Monte-Carlo methods, a photon is propagated through the calculation domain and its scattering, absorption, and re-emission are tracked in detail (Wolf 2003, Wood et al. 2004, Ercolano et al. 2005, Jonsson 2006, Pinte et al. 2006). This allows to treat very complicated spatial distributions (Juvela \& Padoan(2003)), arbitrary scattering functions (Mattila(1970), Witt \& Stephens(1974)), and polarization (Whitney et al. 2003, Bethell et al. 2007). Monte-Carlo methods encountered difficulties when covering re-emission in all directions over many events, and for very small or very high optical depths (Juvela 2005). Meanwhile, several algorithms have been proposed to deal with high optical depths. The major drawback, however, is that there is no global error control when using MonteCarlo schemes.

Ray-tracing solvers can treat arbitrary density configuration with full error control. General purpose solvers for ordinary differential equations can be used to overcome the problem of strongly varying optical depth when using the ray-tracing solution method. These schemes are available in high-order accuracy and with adaptive step size control (e.g. advanced 5th-order Runge Kutta solvers). But they require the implicit recalculation of the step size and become very time-consuming when the optical depth is high, so that more sophisticated solvers are required.

Grid-based solvers, in combination either with finite differencing or short characteristics, have the advantage of error control on the grid. The drawback is the stiff grid so that the resolution of complex 3D structures needs an appropriate grid generation algorithm (Steinacker et al. 2003). Adaptive grids became standard in lower-dimensional problems like hydrodynamical calculations - for RT, the refinement criterion is less clear. An interpolation between the grids for the temperature iteration is numerically costly though and gives rise to interpolation errors in the obtained solution. In most papers presenting 
grid-based 3D RT codes, numerical diffusion has not been considered and taken into account (Steinacker et al. 2002a). The effect is well-known in the course of discretizating hyperbolic equations.

Moment methods are well-posed to treat the optically thick regime and are usually applied to radiation fields with a moderately varying direction dependency. They encounter problems when describing a strongly peaked radiation field arising in the parts of the computational domain where the optical depths is of the order of unity.

Further methods are now in use based on triangulation, or hybrid approaches combining methods (a collection of approaches can be found under www.mpia.de/RT08/).

Radiative transfer on adaptively refined grids. With a finite differencing scheme, the ray equation can be discretized. But the linear grids will not work to resolve the large density gradients. We have proposed to calculate an adaptively refined grid for each wavelength separately as the optical depths strongly varies with wavelength (Steinacker et al. 2002b). We have generated grids which are minimizing the 1st-order discretization error in the scattered radiation intensity and provided global error control for solutions of RT problems on this grid. In order to reduce the grid point number in regions where the optical depth becomes large we have proposed to use the concept of penetration depth. The proposed grid generation algorithm is easy to implement, allows pre-calculation of the grids and storage in integer arrays, making a fast solution of the 3D RT equation possible. The drawback of this method is the cost of interpolation calculations and the introduced interpolation errors when it comes to calculating the temperatures using the different grids. Ray-tracing through regions of very high optical depth. Especially in massive star formation, the optical depth can vary 6 orders of magnitude or more (Beuther \& Steinacker 2007). Commonly, most solvers, be it a Monte-Carlo approach or ray-tracing, are forced to perform small steps slowing down the code. A new ray-tracing technique has been proposed by Steinacker et al. (2006) to use the optically thick approximation in order to speed up the calculations. Assuming radial powerlaw dependencies for the density and temperature distribution, we have calculated the absolute solution errors, the numerical effort, and the stepsize variation for a given accuracy. We have shown that advanced ordinary partial equation solvers like 5th-order Runge Kutta with adaptive stepsize control are too expensive to be applied to the inverse 3D RT modeling problem. Instead we suggested a 2nd-order ray-tracing scheme controlling the relative change of the intensity and making use of the diffusion approximation in the regions of high optical depth. The method is designed to cross optical thick regions quickly, to resolve the important regions with an optical depth around unity, and to have a moderate computational expense mandatory for inverse 3D RT modeling. We apply the method to calculate a farinfrared image of a dense molecular cloud core which represents the initial configuration of a star formation process with speed-ups of the order of several hundreds.

\section{Applications}

We illustrate the requirements and capabilities of RT using a few recent applications. Dust Absorption and Emission: An evolving molecular cloud core seen in space. The results of cloud core collapse simulations have been analyzed with RT in Steinacker et al. (2004). In the simulations, it was assumed that initially low-mass condensations pass through a stage of turbulence-dominated condensation where they accumulate mass and merge together to form extended prestellar core-like objects. The typical density structures in the cores were non-spherical throughout their evolution. Using a 3D continuum RT program, images at $7 \mu \mathrm{m}, 15 \mu \mathrm{m}, 175 \mu \mathrm{m}$, and $1.3 \mathrm{~mm}$ were generated for different evolutionary times and viewing angles. As an example, Fig. 1 shows images at these wavelengths for an evolution time of 56000 years after the onset of the gravitation. It 


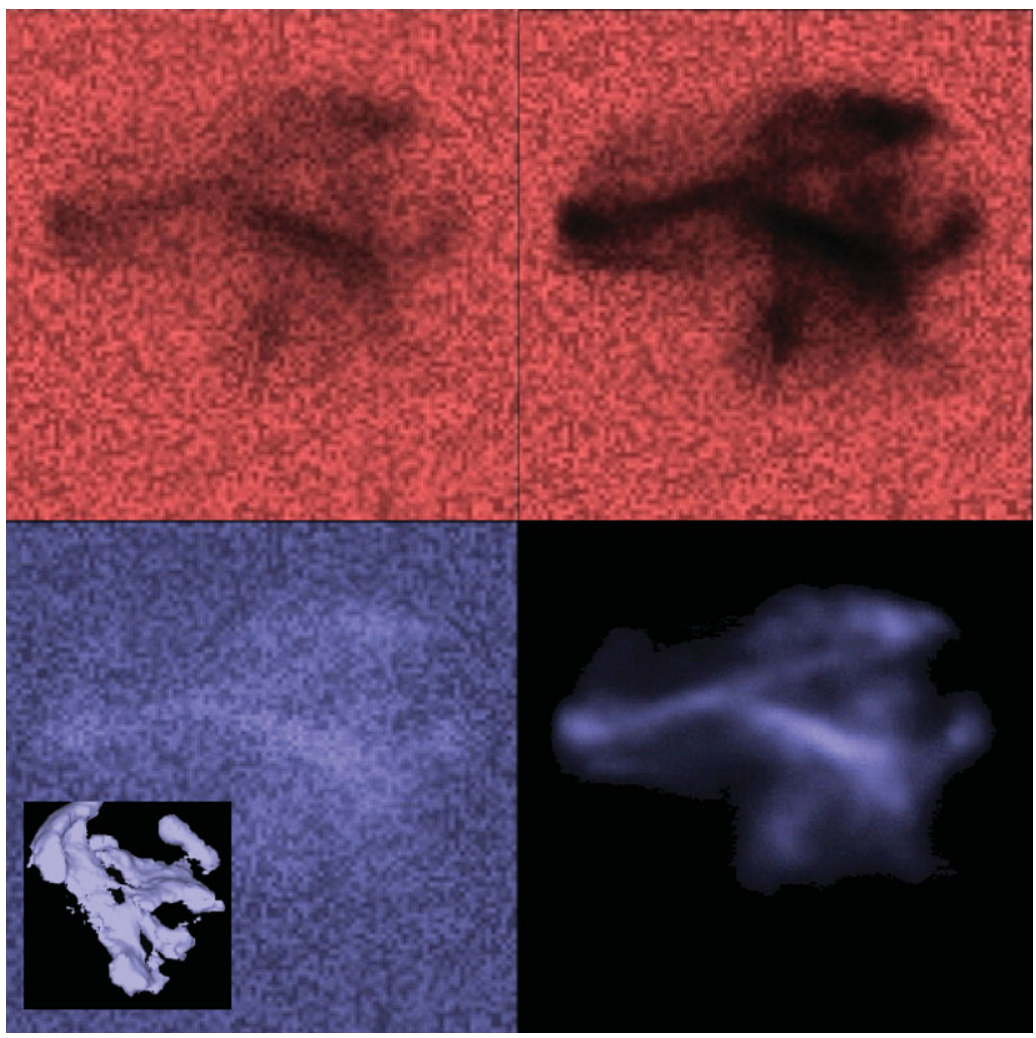

Figure 1. Images of the cloud core fragment at the wavelengths 7 (top left), 15, 175, and $1300 \mu \mathrm{m}$ (bottom right), at a simulation time of 56000 years after the start of the start of the gravitational influence. The inlet shows the iso-density surfaces of the used averaged SPH density distributions of the cloud core fragment corresponding to a density of $4 \times 10^{-17} \mathrm{~kg} \mathrm{~m}^{-3}$. (from Steinacker et al. (2004))

was shown that projection effects can lead to a severe misinterpretation of images: A 1D analysis of the vicinity of the density maxima would suggest density profiles in agreement with 1D-core collapse models. The underlying density structure, however, is intrinsically $3 \mathrm{D}$ and deviates strongly from the obtained 1D model distribution.

The massive disk candidate SO1 in M1\%. While observations show that young lowand intermediate-mass stars are surrounded by a circumstellar disk which are often actively accreting matter from it, only a few candidates for such a circumstellar disk around a massive star are known (see Chini et al. 2004 for a list of candidates).

Steinacker et al. (2006) have analyzed the prominent silhouette structure in M 17 showing a symmetric large-scale pattern in absorption against a bright background, with a central emission region, an hourglass-shaped reflection nebula perpendicular to the extinction bar, a complex outflow over and below the dark extinction lane, and signatures for accretion of matter. Due to the large scale and the strong symmetry of the structure, as well as its presence within a massive star formation region, it attracts special attention as a candidate for a massive disk around a star that might be massive or has the potential to reach such a mass. While the estimate of the disk mass for most massive disk candidates comes from low resolution FIR/mm measurements, they used the advantage that due to the background illumination, the column density can be determined at $\lambda=2.2 \mu \mathrm{m}$ from a high-resolution NAOS/CONICA image. 


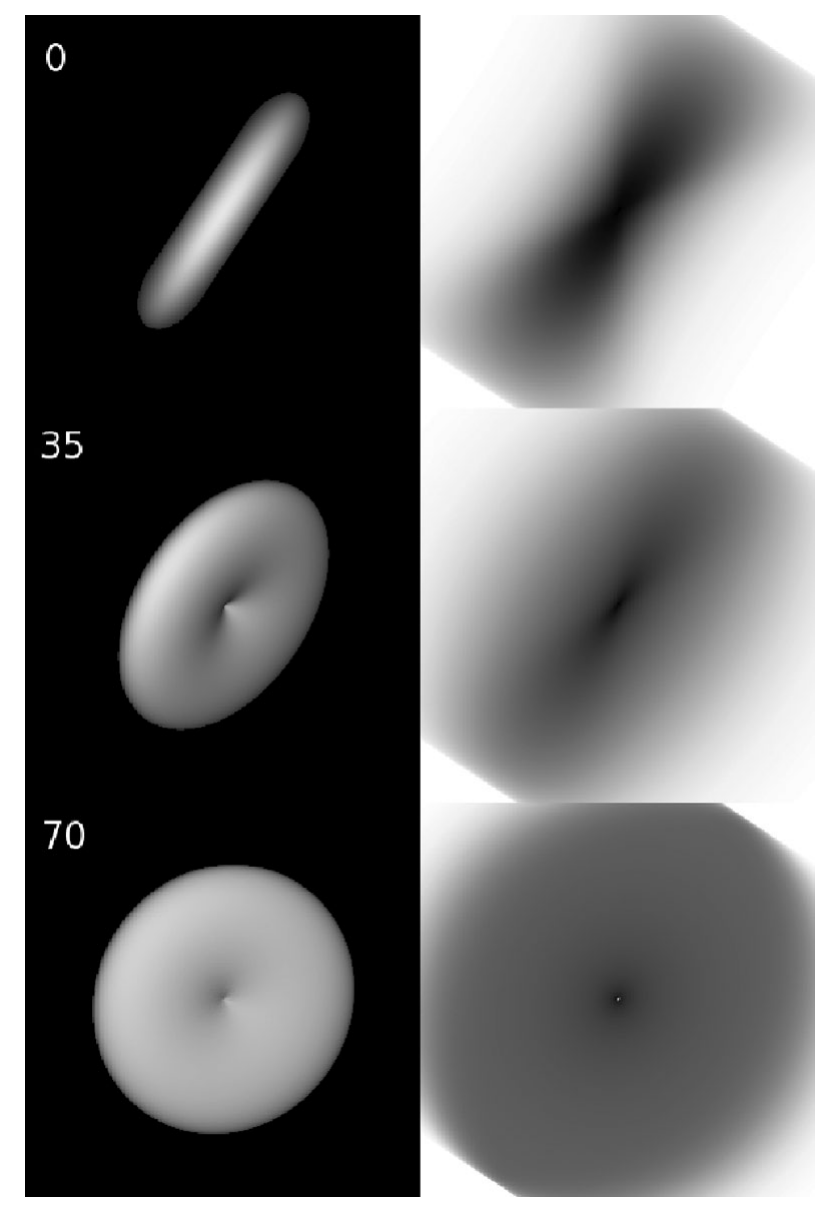

Figure 2. Theoretical absorption image of the circumstellar disk using the optimized fit parameters and varying the inclination angle against the edge-on case. The left panel shows an iso-density surface of the disk distribution (the inclination angle against the edge-on case is given in degrees). The right panel shows the corresponding $2.2 \mu \mathrm{m}$ absorption image . (from Steinacker et al. (2006))

It was investigated whether the observed extinction structure is consistent with a model of a circumstellar rotationally-symmetric flattened density distribution. Applying a commonly used analytical disk model with a powerlaw in radius and a vertical Gaussian distribution, they have fitted a 7 parameter model to the about 8000 pixels of the image. The PSF of a point source was covered by about 3.3 pixels, corresponding to about 2400 independent data points. It was found that the derived optical depth is consistent with a rotationally-symmetric distribution of gas and dust around the central emission peak. The extent of the axisymmetric disk part is about $3000 \mathrm{AU}$, with a warped pointsymmetrical extension beyond that radius, and therefore larger than any circumstellar disk detected sofar. The resulting theoretical absorption image of the disk is shown in Fig. 2 for different inclination angles, along with the corresponding $2.2 \mu \mathrm{m}$-image.

The mass of the entire disk estimated from the column density was discussed depending on the assumed distance and the dust model and ranges between 0.02 and $5 M_{\odot}$. The derived disk mass range is smaller than the mass range of 110 to $330 M_{\odot}$ derived from ${ }^{1} 3 \mathrm{CO}$ data (Chini et al. 2004). 


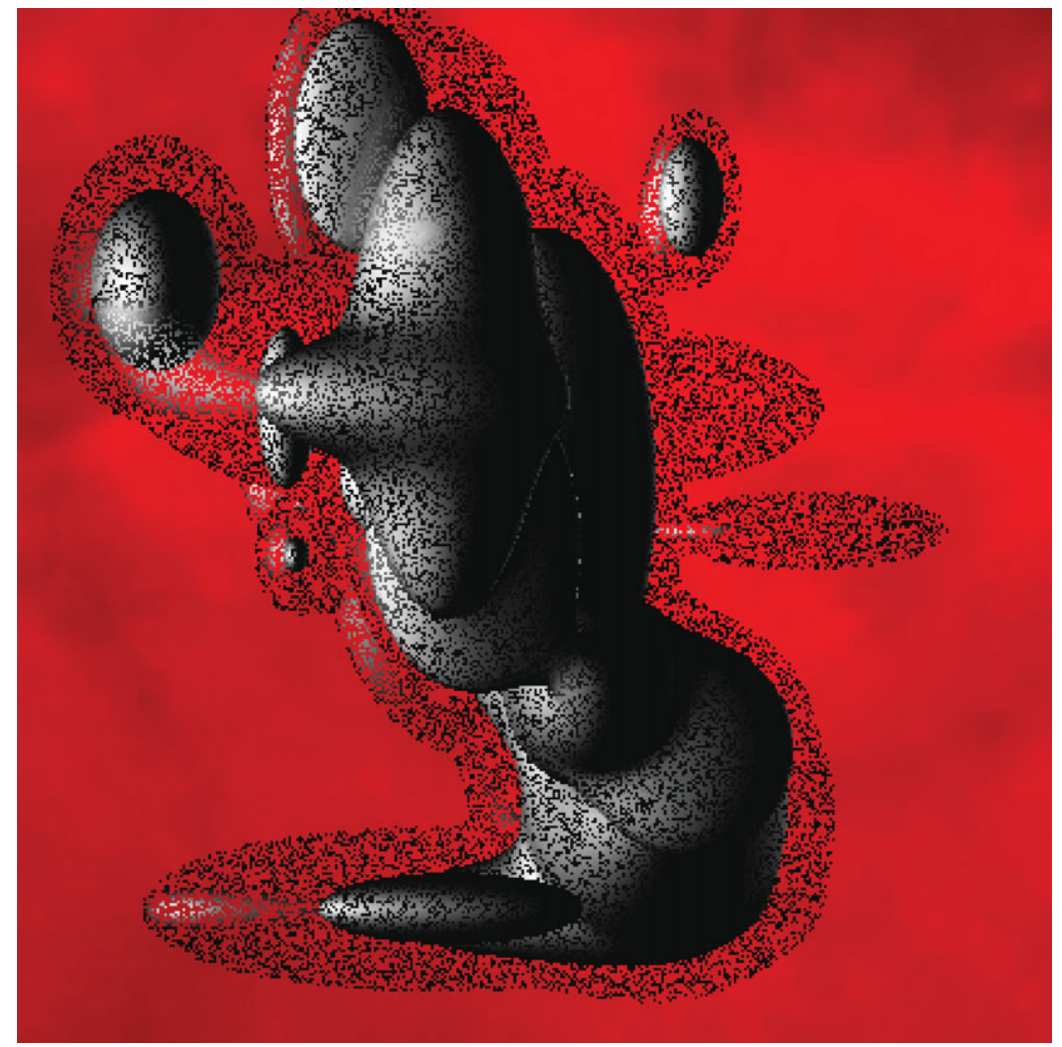

Figure 3. Two layers of constant density (solid and semi-transparent) illustrating of the full 3D configuration of the molecular cloud core Rho Oph D. The density was determined by fitting a continuum map at $1.3 \mathrm{~mm}$ and two continuum maps at 7 and 15 micron. (from Steinacker et al. (2005))

The next step: Inverse 3D RT modeling of the molecular cloud core Rho Oph D. Calculating images from a given density and temperature distribution using a RT code can quickly reach the capabilities of current computers. The finally desired approach would be, however, to determine the density and temperature structure in $3 \mathrm{D}$ from the images. This so-called inverse RT problem features several difficulties, most prominently the loss of information due to the line-of-sight integration performed during the observations. It was shown in Steinacker et al. (2005) that inverse RT transfer is possible nevertheless already for the analysis of molecular cloud cores. A new method was proposed to model the 3D dust density and temperature structure of such a cores. It is based on the fits of multi-wavelengths continuum images in which the core is seen in absorption and emission and requires only a few computations with a 3D CRT code. The large parameter space of a 3D structure is covered using simulated annealing as optimization algorithm.

The method was applied to model the dense molecular cloud core $\rho$ Oph D. In the MIR, the core is seen in absorption against a bright background from the photo-dissociation region of the nearby $\mathrm{B} 2 \mathrm{~V}$ star, illuminating the cloud from behind. Two ISOCAM images at 7 and $15 \mu \mathrm{m}$ have been fitted simultaneously by representing the dust distribution in the core with a series of 3D Gaussian density profiles. The background emission behind the core was interpolated from nearby regions of low extinction. Using simulated annealing, a $2 \mathrm{D}$ column density map of the core was obtained. The column density of the core has a complex elongated pattern with two peaks, with the southern peak being more 


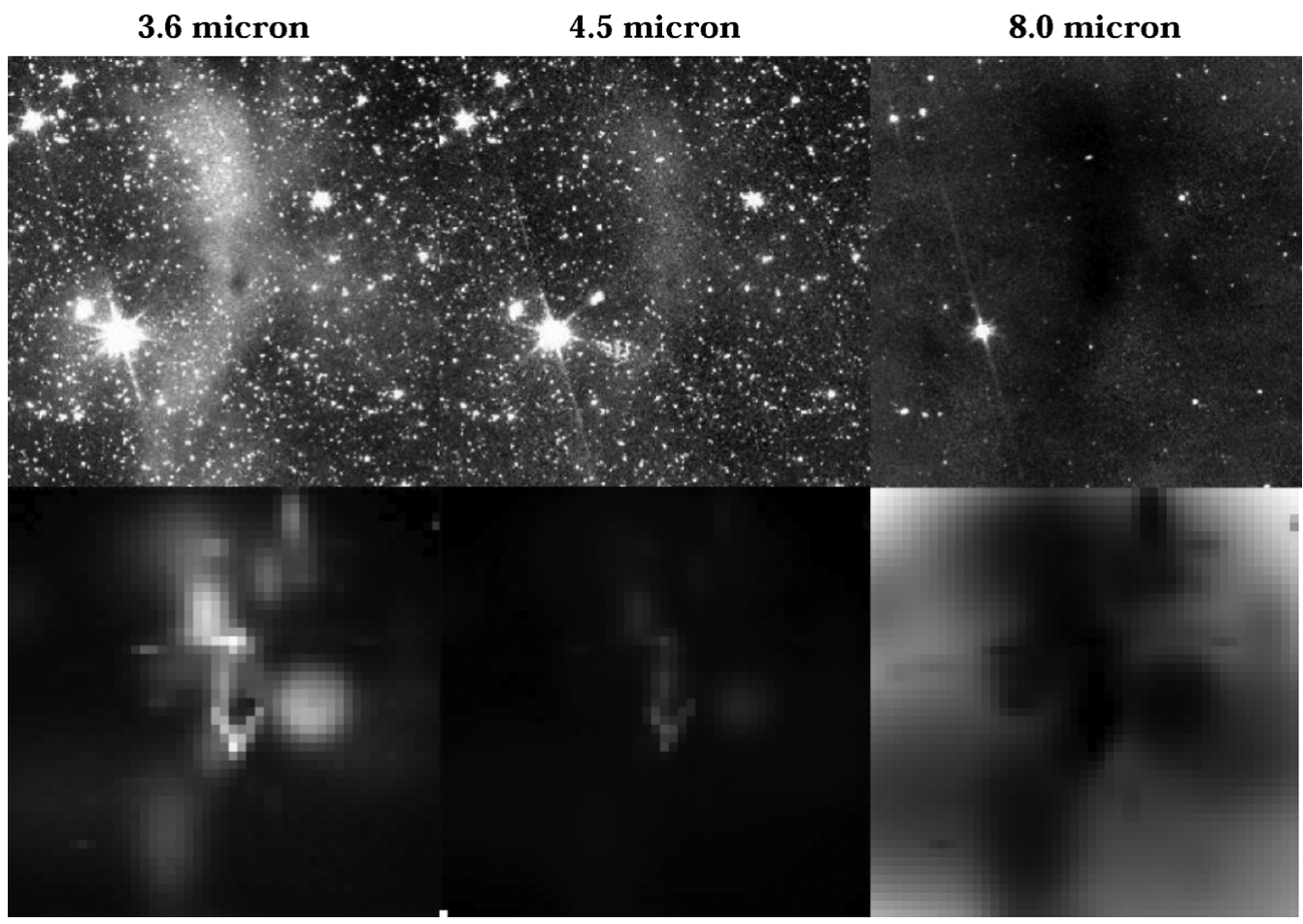

Figure 4. Comparison of the three Spitzer images at 3.6, 4.5, and $8 \mathrm{~m}$ of the inner 66000 AU of L183 (top) with scattered light models based on grains growing as a function of density (bottom). The underlying 3D structure of the model images is consistent with the measured $\mathrm{Av}$ map. The general pattern of the modeled diffuse emission is similar while, the flux is about a factor of 2 lower in the model. (from Steinacker et al. (2010))

compact. Fig. 3 illustrates the resulting 3D density data cube by showing two iso-density layers (semi-transparent and solid).

Coreshine: the new window to explore dust growth in dense molecular cloud cores. Theoretical arguments suggest that dust grains should grow in the dense cold parts of molecular clouds. Evidence of larger grains has so far been gathered in near/mid infrared extinction and millimeter observations. Interpreting the data is, however, aggravated by the complex interplay of density and dust properties (as well as temperature for thermal emission). In Steinacker et al. (2010), new Spitzer data of L183 have been presented in bands that contain substantial and marginal PAH emission. A visual extinction map of L183 was fitted by a series of 3D Gaussian distributions. For different dust models, the scattered MIR radiation images of structures have been calculated that agree with the $A_{\mathrm{V}}$ map and they were compared to the Spitzer data. The Spitzer data of L183 show emission in the 3.6 and $4.5 \mu \mathrm{m}$ bands, while the $5.8 \mu \mathrm{m}$ band shows slight absorption. This emission was interpreted to be MIR scattered light from grains located further inside the core, and was called "coreshine". Models with grains growing with density yield images with a flux and pattern comparable to the Spitzer images in the bands 3.6, 4.5, and $8.0 \mu \mathrm{m}$.

\section{References}

Bethell, T. J., Chepurnov, A., Lazarian, A., \& Kim, J. 2007, ApJ, 663, 1055

Beuther, H. \& Steinacker, J. 2007, ApJL, 656, L85 
Bianchi, S. 2007, A\&\&A, 471, 765

Chini, R., Hoffmeister, V., Kimeswenger, S., Nielbock, M., Nürnberger, D., Schmidtobreick, L., \& Sterzik, M. 2004, Nature, 429, 155

Ercolano, B., Barlow, M. J., \& Storey, P. J. 2005, MNRAS, 362, 1038

Guhathakurta, P. \& Draine, B. T. 1989, ApJ, 345, 230

Jonsson, P. 2006, MNRAS, 372, 2

Juvela, M. \& Padoan, P. 2003, A\&A, 397, 201

Juvela, M. 2005, $A \mathscr{E} A, 440,531$

Mattila, K. 1970, A\&A, 9, 53

Min, M., Hovenier, J. W., \& de Koter, A. 2003, A\& A, 404, 35

Pinte, C., Ménard, F., Duchêne, G. \& Bastien, P. 2006, A\&A, 459, 797

Siebenmorgen, R., Kruegel, E., \& Mathis, J. S. 1992, A\&BA, 266, 501

Steinacker, J., Thamm, E. \& Maier, U. 1996, JQSRT, 97, 56

Steinacker, J., Hackert, R., Steinacker, A., \& Bacmann, A. 2002, JQSRT, 73, 557 (a)

Steinacker, J., Bacmann, A., \& Henning, T. 2002, JQSRT, 75, 765 (b)

Steinacker, J., Henning, T., Bacmann, A., \& Semenov, D. 2003, A\&A A, 401, 405

Steinacker, J., Lang, B., Burkert, A., Bacmann, A., \& Henning, T. 2004, ApJL, 615, L157

Steinacker, J., Bacmann, A., Henning, T., Klessen, R., \& Stickel, M. 2005, A\&\&A, 434, 167

Steinacker, J., Bacmann, A., \& Henning, T. 2006, ApJ, 645, 920

Steinacker, J., Chini, R., Nielbock, M., Nürnberger, D., Hoffmeister, V., Huré, J.-M., \& Semenov, D. 2006, A\&A, 456, 1013

Steinacker, J., Pagani, L., Bacmann, A., \& Guieu, S. 2010, A\&A, 511, A9

Whitney, B. A., Wood, K., Bjorkman, J. E., \& Wolff, M. J. 2003, ApJ, 591, 1049

Witt, A. N. \& Stephens, T. C. 1974, AJ, 79, 948

Wolf, S. 2003, Computer Physics Communications, 150, 99

Wood, K., Mathis, J. S., \& Ercolano, B. 2004, MNRAS, 348, 1337 


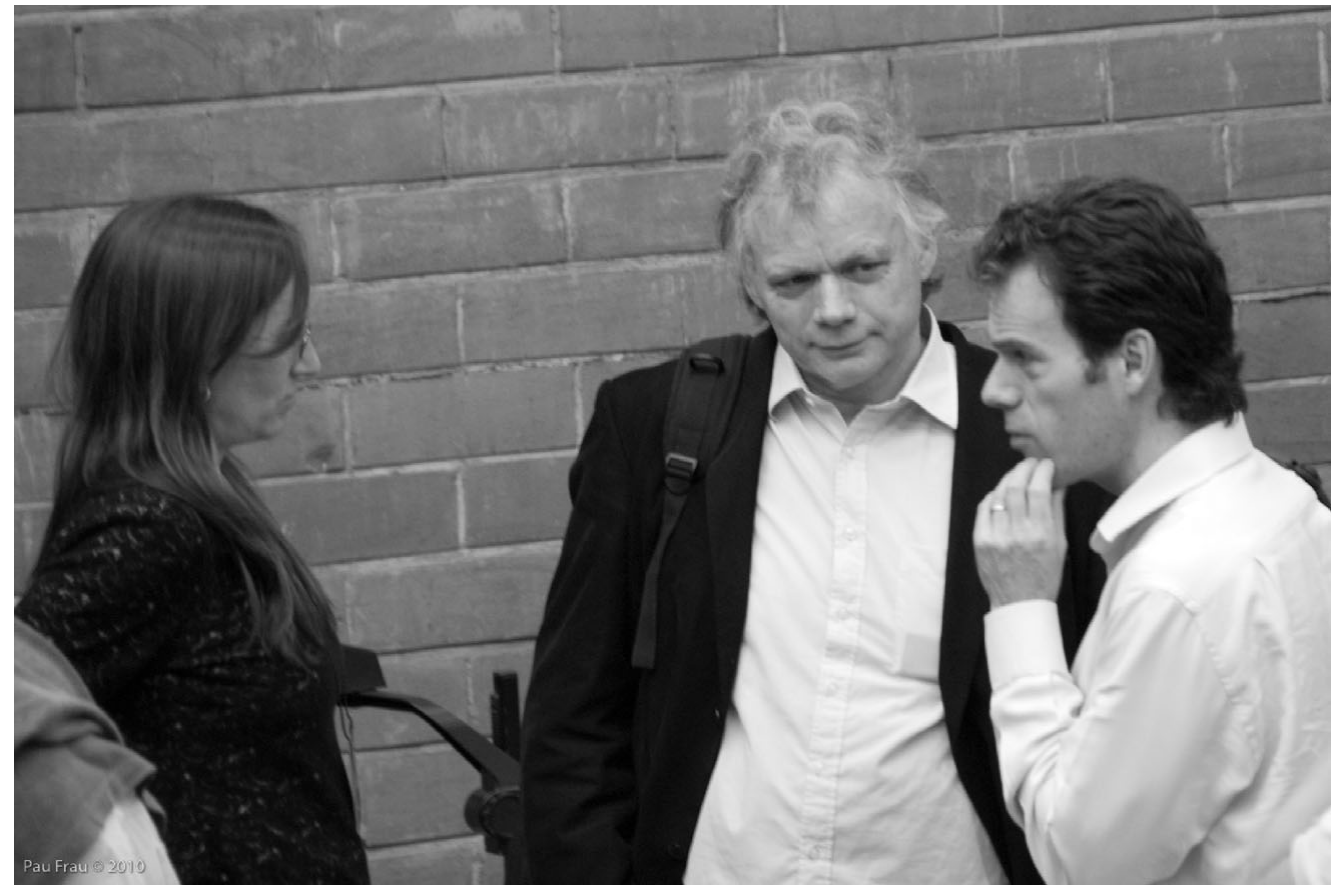

Eve Ostriker, Åke Nordlund, and Paolo Padoan 